\title{
Origami Multistability: From Single Vertices to Metasheets
}

\author{
Scott Waitukaitis, ${ }^{1}$ Rémi Menaut, ${ }^{1,2}$ Bryan Gin-ge Chen, ${ }^{3}$ and Martin van Hecke ${ }^{1}$ \\ ${ }^{1}$ Huygens-Kamerlingh Onnes Lab, Leiden University, P.O. Box 9504, 2300 RA Leiden, Netherlands \\ ${ }^{2}$ École Normale Supérieure de Lyon, Université Claude Bernard Lyon 1, BP 7000, 69342 Lyon Cedex 07, France \\ ${ }^{3}$ Instituut-Lorentz for Theoretical Physics, Leiden University, P.O. Box 9506, 2333 CA Leiden, Netherlands \\ (Received 7 September 2014; revised manuscript received 17 October 2014; published 4 February 2015)
}

\begin{abstract}
We show that the simplest building blocks of origami-based materials—rigid, degree-four vertices—are generically multistable. The existence of two distinct branches of folding motion emerging from the flat state suggests at least bistability, but we show how nonlinearities in the folding motions allow generic vertex geometries to have as many as five stable states. In special geometries with collinear folds and symmetry, more branches emerge leading to as many as six stable states. Tuning the fold energy parameters, we show how monostability is also possible. Finally, we show how to program the stability features of a single vertex into a periodic fold tessellation. The resulting metasheets provide a previously unanticipated functionality — tunable and switchable shape and size via multistability.
\end{abstract}

Mechanical metamaterials are elastic media with extraordinary properties that arise from their microstructure [1-15]. Currently established functionalities include negative Poisson's ratio [2], vanishing shear modulus [3-5], negative compressibility [6,7], pattern transformation [8-12], switchable multistability [13], and topological insulation $[14,15]$. The building blocks for these materials are typically quasi-1D rods or springs, but recently, origami-inspired metamaterials made from folding planar structures have gained interest. This represents an important departure for a variety of reasons. First, the deformations of folding-based materials can be highly nonlinear owing to the complex constraint space imposed by the fold network. Second, their energetic landscapes do not arise from central-force linear springs but instead through torsional spring interactions [16-19].

Most recent attention has been focused on the Miura-Ori, a fold tessellation well known for its negative Poisson's ratio. Silverberg et al. recently used Miura-Ori to create a metamaterial with tunable stiffness by introducing a reversible "pop-through" defect [18]. This local defect, permitted via plate bending, is one of a few specific examples of bistability in folding planar structures-others include the symmetric water bomb vertex [20] and the hypar [21]. Such multistability is a desirable property for the design of metamaterials as it allows reprogrammable reconfiguration of shape and bulk properties.

Here, we reveal how folding planar structures offer a platform for globally multistable metamaterials-structures capable of multiple stable shapes and sizes. Our building block is the degree-four vertex, i.e., four rigid plates connected by four folds (or hinges) that meet at a point. This is the simplest building block for origami metamaterials because it is a one-degree-of-freedom mechanism (lower $n$-vertices are rigid [22]). We show that the interesting physical properties arise from complexity in the physical configuration space, to which we now turn our attention.

Generic configuration space.-We first consider generic four-vertices, i.e., those without collinear folds, symmetry, or flat foldability [23]. We specify the flat-state geometry by the set of sector angles $\left\{\alpha_{i}\right\}$, where each $\alpha_{i}<\pi$ and $\Sigma_{i} \alpha_{i}=2 \pi$ [Fig. 1(a)]. A folded state is described by the folding angles $\left\{\rho_{i}\right\}$, the complements of the dihedral angles between plates $i$ and $i-1$ (cyclic permutations understood). We take positive folding angles as "valleys" and negative ones as "mountains." We begin by considering three basic questions: (i) What are the possible mountainvalley assignments for the folds? (ii) Which folds can be maximally folded to $\pm \pi$ ? (iii) What are the relationships between the folding angles? Below, we summarize the answers; a detailed treatment, including nongeneric folds and nonflat paper, is in preparation [24].

(i) Huffman noted that one folding angle must have the opposite sign from the rest [25]. Which folds can do this? Figure 1(c) shows two mountain-valley assignments for the vertex shown in Fig. 1(a). In each case, the "unique" fold with the sign opposite from the rest is cupped inside the others. This implies that fold $j$ can be unique if

$$
\alpha_{j-1}+\alpha_{j} \leq \pi,
$$

where equality corresponds to a nongeneric case [24]. For generic vertices, it immediately follows that two folds are unique and straddle a common plate. This implies that generic four-vertices have two branches of motion that intersect at the flat state-without losing generality, we label the unique folds 1 and 2 and the respective branches for which they are unique I and II.

(ii) A vertex "binds" when one fold (or more) reaches $\pm \pi$ and prevents further motion; bound states therefore 

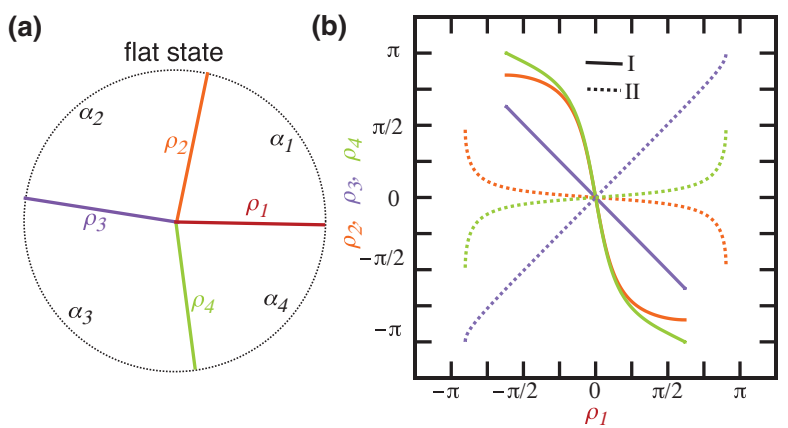

(c)

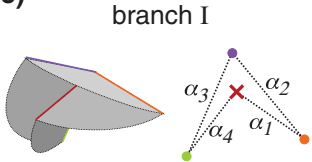

branch II

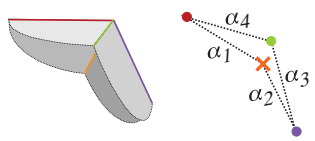

(d)
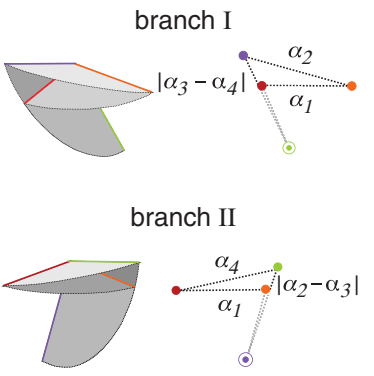

FIG. 1 (color). Configuration space. (a) The flat state is defined by sector angles $\left\{\alpha_{i}\right\}$ (here, $\left\{\alpha_{i}\right\}=\{1.4,1.6,1.9,2 \pi-4.9\}$ ) and a folded state by folding angles $\left\{\rho_{i}\right\}$. (b) Configuration curves $\rho_{2,3,4}$ vs $\rho_{1}$ for branches I and II. (c) 3D renderings and schematic side views for folding motions on branch I and II (crosses designate unique folds). (d) 3D renderings and schematic side views for bindings on each branch (circled dots designate binding folds). For movies illustrating the folding motions of each branch, see Ref. [26].

determine the ranges of the folding angles. Figure 1(d) shows the vertex from Fig. 1(a) in two bindings. Fold $j$ binding creates a spherical triangle of sides $\left|\alpha_{j-1}-\alpha_{j}\right|$, $\alpha_{j+1}$, and $\alpha_{j+2}$ that must satisfy all three permutations of the spherical triangle inequality. Conveniently, these can be reduced to

$$
\left|\alpha_{j-1}-\alpha_{j}\right| \geq\left|\alpha_{j+1}-\alpha_{j+2}\right|,
$$

where again equality corresponds to a nongeneric case. Generic vertices have two binding folds that straddle a common plate, and, once identified, the values of the remaining folding angles at binding can be calculated with spherical trigonometry [24].

(iii) As four-vertices have just one continuous degree of freedom, we can pick one folding angle to parametrize the others (and later, the energy). Huffman found implicit relationships between these angles [25], but we have derived explicit configuration equations (which are also valid for nonflat paper-see Ref. [24]). We choose $\rho_{1}$ as our parametrizing variable, and in Fig. 1(b), we give example curves for the vertex shown in Fig. 1(a). These are representative of generic vertices in that they are antisymmetric, monotonic, and nonlinear.
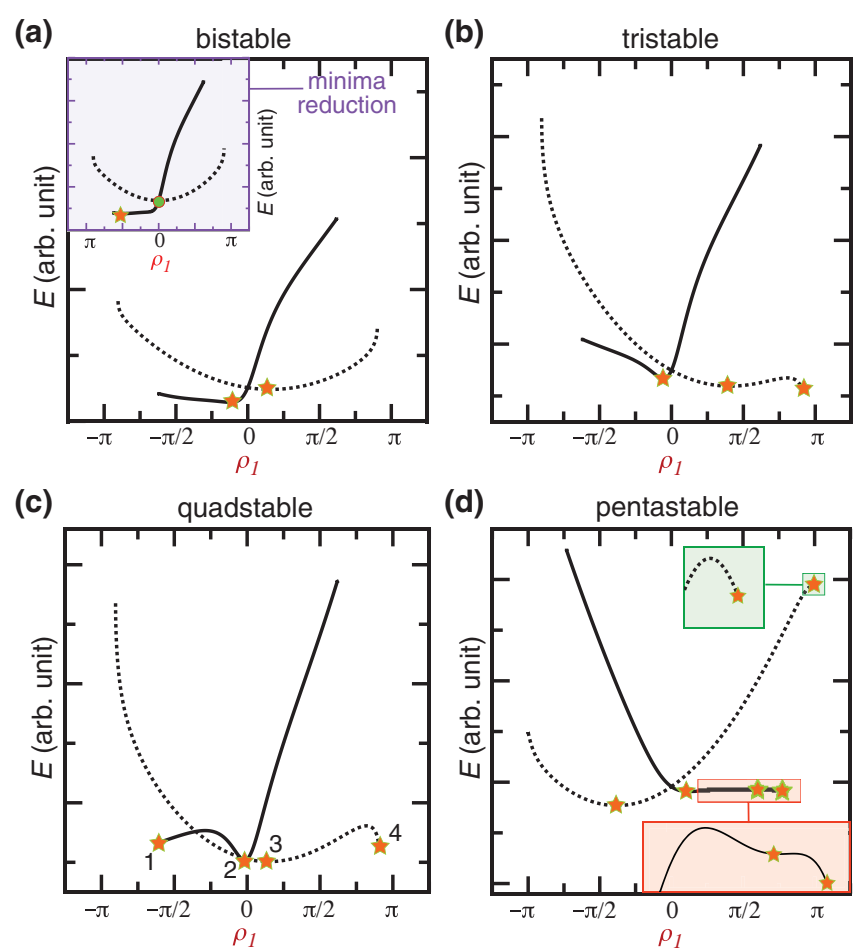

(d) pentastable

(e)
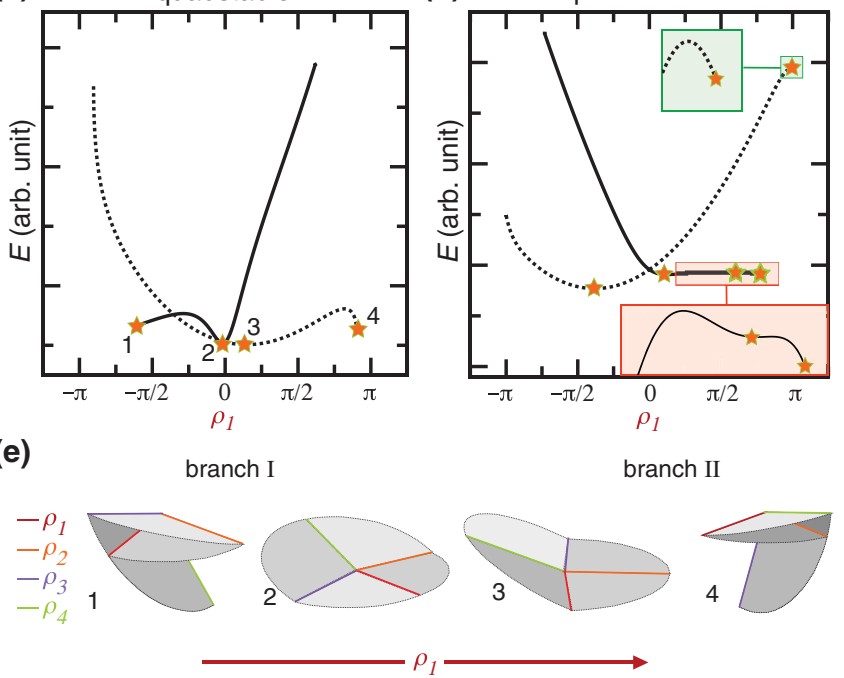

FIG. 2 (color). Example (a) bistable, (b) tristable, (c) quadstable, and (d) pentastable generic vertices. The inset to (a) is a monostable vertex created via minima reduction, while the insets to (d) show zoomed views of the shallow minima. For (a)-(c), $\left\{\alpha_{i}\right\}$ are the same as in Fig. $1(\mathrm{~d})$, all $\kappa_{i}=1$, and the $\left\{\bar{\rho}_{i}\right\}$ are $\{-1.2,1.8,2.3,0.5\}$ and $\{-2.235 \ldots, 1.8,2.3,0.5\}$ for (a) and its inset, $\{-0.1,-1.5,2.2,2.8\}$ for (b), and $\{-2.6,-2.9,3.1,2.4\}$ for (c). The rare pentastable vertex has different parameters: $\left\{\alpha_{i}\right\}=\{1.1,2.0,1.9,2 \pi-5.0\},\left\{k_{i}\right\}=\{0.3,0.1,1.0,0.6\}$, and $\left\{\bar{\rho}_{i}\right\}=\{2.7,-2.1,-2.4,1.4\}$. (e) Pictures of the four stable states of the quadstable landscape of (c), as indicated.

Energy landscape and multistability.-We model the vertex energy with torsional springs in the folds

$$
E_{V}=\frac{1}{2} \sum_{i=1}^{4} \kappa_{i}\left(\rho_{i}-\bar{\rho}_{i}\right)^{2}
$$

where $\left\{\kappa_{i}\right\}$ are spring constants $\left(0 \leq \kappa_{i} \leq 1\right)$ and $\left\{\bar{\rho}_{i}\right\}$ are rest angles $\left(-\pi \leq \bar{\rho}_{i} \leq \pi\right)$. This form is both simple [16,27] and experimentally valid $[20,28]$ and most importantly allows for frustration when $\left\{\bar{\rho}_{i}\right\}$ does not reside on any of the folding branches. The branching leads to two energy curves; thus, the extreme value theorem suggests at least bistability-one minimum per branch, as in Fig. 2(a). However, as Figs. 2(b)-2(d) show, it is possible to have 
more than one minimum per branch, leading to tri-, quad-, and even pentastable vertices. We remark that variation in the $\left\{\kappa_{i}\right\}$ adds extra tunability but is not crucial: The bi-, tri-, and quadstable examples shown in Figs. 2(a)-2(c) were created with equal strength springs and changing only the rest angles. The rare vertex with five minima was so far only found for unequal spring constants.

To understand why multiple minima per branch occur, first note that surfaces of constant energy in the 4D space of folding angles are ellipsoidal shells centered at the global minimum $\left\{\rho_{i}\right\}=\left\{\bar{\rho}_{i}\right\}$. Physically realizable minima occur at points where the $1 \mathrm{D}$ configuration curves are tangent to one of the ellipsoidal shells, and this can happen in multiple locations if the curves move both toward and then away from the global minimum [29]. Hence, multiple minima on a single branch arise from the nonlinearity in the configuration curves in conjunction with the general inaccessibility of the global minimum, i.e., frustration.

The complexity of the configuration equations prevents us from predicting the number and location of minima analytically. Instead, we do this numerically and discover possible stability landscapes by uniformly sampling the accessible space of $\left\{\alpha_{i}\right\},\left\{\kappa_{i}\right\}$, and $\left\{\bar{\rho}_{i}\right\}$. The results for generic vertices, summarized in Table I, reveal that bistable arrangements occupy the vast majority of the phase space, followed by a much smaller fraction of tristable vertices, an even smaller fraction of quadstable ones, and very rare pentastable vertices [30]. As we will show, special vertices with symmetry allow up to six stable states, and the presence of the pentastable vertex leads us to speculate that generic vertices might be capable of six minima as well - three per branch. However, the trends in Table I make it clear that the likelihood of generating such a vertex from random sampling is incredibly small.

Monostable vertices are possible but lie in a lower dimensional subset of measure 0 and are not encountered in our sampling. To create such a vertex, we eliminate one minimum by moving it to a branching point, e.g., the flat state. In the inset to Fig. 2(a), we show an example where we have tuned the parameters of a bistable vertex to have its branch II minimum at the flat state (note the similarity in the energy curves). A vertex drawn to such a

TABLE I. Multistability probabilities. Values calculated in each case from $10^{6}$ instances with uniformly sampled $\left\{\alpha_{i}\right\}$, $\left\{\kappa_{i}\right\}$, and $\left\{\bar{\rho}_{i}\right\}$.

\begin{tabular}{lcccccc}
\hline \hline Geometry & 1 & 2 & 3 & 4 & 5 & 6 \\
\hline Generic & 0 & 0.9311 & 0.0657 & 0.0032 & $10^{-6}$ & 0 \\
Flat foldable & 0 & 0.9418 & 0.0574 & 0.0008 & 0 & 0 \\
Single collinear & 0 & 0.9710 & 0.0290 & 0 & 0 & 0 \\
Double collinear & 0 & 1 & 0 & 0 & 0 & 0 \\
Single symmetric & 0 & 0 & 0 & 0.9768 & 0.0232 & 0 \\
Double symmetric & 0 & 0 & 0 & 0 & 0 & 1 \\
\hline \hline
\end{tabular}

branching-point minimum will proceed to lower its energy by going to a minimum on the other branch, and, as most generic vertices are bistable, such carefully tuned vertices will typically be monostable. Conditions that guarantee this on a particular branch can be found by linearly expanding its configuration equations near the branching point, using them in the energy expression, and then setting its derivative to 0 (see Ref. [24] for full details).

Nongeneric vertices.-We now turn our attention to nongeneric vertices, i.e., ones that are flat foldable, have collinear folds, and/or have additional symmetries. These properties influence the multiplicity and nonlinearity of the branches and therefore have strong effects on the possible number of stable states.

First, flat-foldable vertices are ones whose bound states are flat, i.e., those for which all folding angles at binding are $\pm \pi$. Historically, most of the attention from mathematicians and physicists studying origami has been focused on flatfoldable vertices. Within our general framework, a vertex will fold flat if it meets the Kawasaki-Justin condition [23]; i.e., the sum of alternating $\alpha_{i}$ is equal to $\pi$. Generic flatfoldable vertices still have two branches, and their configuration curves are still nonlinear; thus, landscapes beyond bistability are possible. Table I shows that flat-foldable vertices significantly suppress the likelihood of having more than two minima. We understand this from the observation that the configuration curves of flat-foldable vertices are, in general, less curved than those of generic vertices.

Second, single collinear vertices have two opposing folds aligned, as shown in Fig. 3(a) for folds 1 and 3. Here, branch II becomes strictly linear, as in Fig. 3(e), so that the energy is simply quadratic in $\rho_{1}$ and yields just
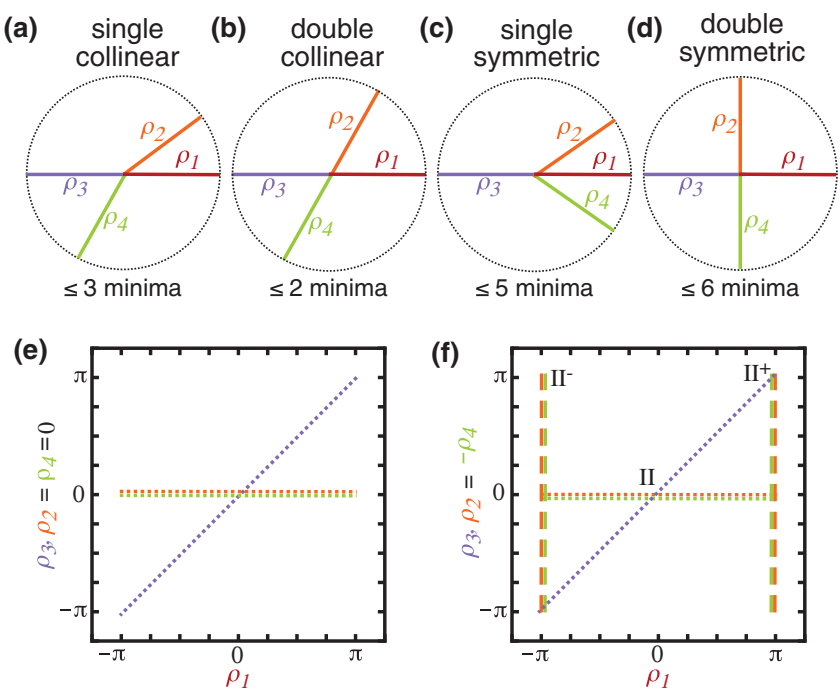

FIG. 3 (color). (a)-(d) Four types of special vertices. (e) Linear branch II curves of $\rho_{2,3,4}$ vs $\rho_{1}$ for a single collinear vertex with folds 1 and 3 collinear, as in (a). (f) Linear curves for branches II, $\mathrm{II}^{-}$, and $\mathrm{II}^{+}$for a single symmetric vertex with folds 1 and 3 collinear, as in (c). 
one minimum. Branch I remains nonlinear and can still have multiple minima. As a consequence, single collinear vertices are more likely to have fewer minima than generic ones (in our sampling, we find at most three-see Table I). For a double collinear vertex [where both sets of folds are collinear but without reflection symmetry across foldsFig. 3(b)], both branches are strictly linear and such vertices are generically bistable unless there is minima reduction.

Third, new branches emerge when there is reflection symmetry across collinear folds [Fig. 3(c)]. For a single symmetric vertex with folds 1 and 3 collinear, we see that when they bind, subsequent folding of folds 2 and 4 becomes possible. We designate these new branches as $\mathrm{II}^{-}$and $\mathrm{II}^{+}$ (corresponding to the bindings at $\rho_{1}=\rho_{3}=\mp \pi$, respectively). These branches are linear, as shown in Fig. 3(f). As with single collinear vertices, branch I remains normal and can still produce multiple minima. In random sampling, we find at most five minima (up to one for each linear branch and two for the normal one), but four minima is the most likely outcome (Table I). For double symmetry [Fig. 3(d)], there are six linear branches and such vertices have six minima unless there is minima reduction (Table I).

Metasheets.-To create multistable metasheets, we take a base four-vertex that is already multistable and build flat-state tiles by drawing parallelograms from neighboring folds, as in Fig. 4(a). The resulting tessellation introduces three new vertices (a rotated original vertex,

(a)
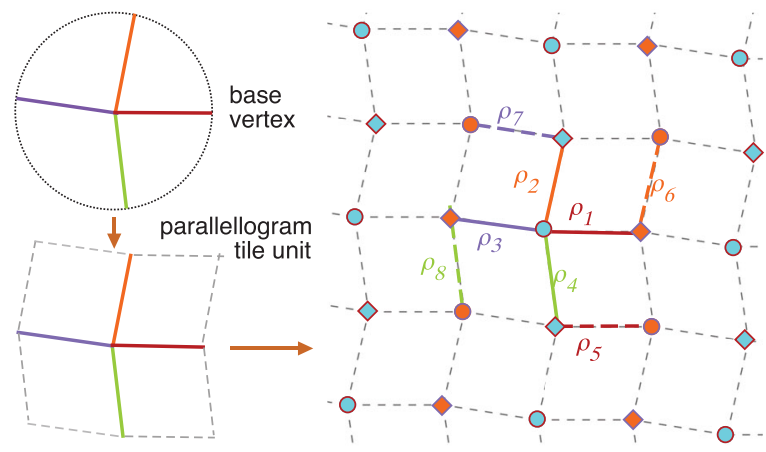

(b) branch I
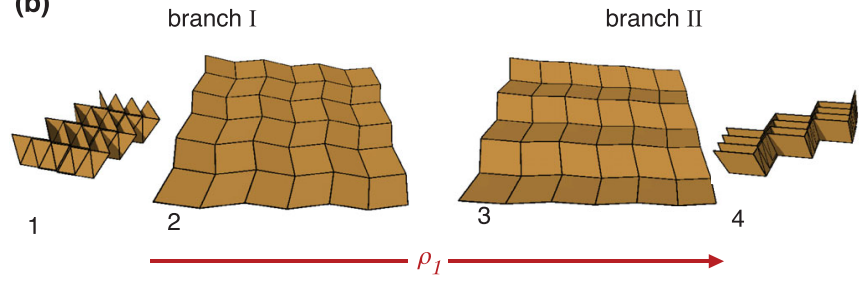

FIG. 4 (color). Metasheets. (a) Procedure to tile an arbitrary four-vertex. The tiling consists of the original vertex (cyan circles), the rotated original vertex (orange circles), the complementary vertex (cyan diamonds), and the rotated complementary vertex (orange diamonds). Homogeneous states are completely described by the folding angles $\rho_{1 \rightarrow 8}$. (b) The four homogeneous stable states of a metasheet with the same parameters as Fig. 2(c). For movies, see Ref. [26]. a "complementary vertex" with sector angles $\left\{\pi-\alpha_{i}\right\}$, and a rotated complementary vertex), but the unique folds, binding folds, and binding angles of the original vertex are unchanged and the sheet remains a one-degree-of-freedom mechanism. While the original vertex had four folding angles, homogeneous states of the tiling have eight. Via reflection symmetry, such states have $\rho_{i+4}=-\rho_{i}$. By choosing $k_{i+4}=k_{i}$ and $\bar{\rho}_{i+4}=-\bar{\rho}_{i}$, we create a simple relationship between the sheet and base vertex energies

$$
E_{T}=\frac{N}{2} \sum_{i=1}^{4} \kappa_{i}\left[\left(\rho_{i}-\bar{\rho}_{i}\right)^{2}+\left(-\rho_{i}+\bar{\rho}_{i}\right)^{2}\right]=2 N E_{V}
$$

where $N$ is the number of tiles in the tessellation.

In Fig. 4, we show images of the four minima states of a metasheet with the quadstable energy landscape of Fig. 2(c). One particularly striking feature is the ridge patterning, which changes from vertical to horizontal as the branches are switched. It is easy to imagine that switching between such horizontal or vertical polarizations could be useful in micromechanical devices or optical elements such as diffraction gratings. (See the Supplemental Material [26] for a movie illustrating the pattern transformation.)

We can now explain why such global multistability has never been encountered previously. As we mentioned, most origami metamaterials thus far have been based on the Miura-Ori tessellation $[16,18,20]$. Following our tiling procedure, such a pattern is made using a single symmetric vertex base, just as in Fig. 3(c). While this geometry creates the potential for several minima (up to five in our phase space search), simplifying assumptions have hidden this functionality. In particular, all studies to date have assumed that (1) $\kappa_{1}=\kappa_{3}$ and $\kappa_{2}=\kappa_{4}$ and (2) that the global minimum lies on the normal branch. These assumptions conspire to place the minima of each linear branch at its branching point (leading to three minima reductions). The position of the global minimum on the normal branch prevents a second minimum there because the configuration curves are monotonic. As a result, such systems have perhaps given the impression that monostability is the norm when in fact it is an exception.

Discussion and outlook.-We have shown that folding principles offer a platform to create shape-reconfigurable metamaterials. This begins with the simplest possible building block, the rigid four-vertex, which has two branches of folding motion emerging from the flat state. Naïvely, one might expect generic four-vertices to be bistable (one minimum per branch), but nonlinear relationships between folding angles lead to complex energy landscapes with as many as five minima. Collinear folds and symmetries change this by making branches linear and/ or adding new branches, creating the possibility for up to six minima. By precisely tuning the fold energy parameters, we have shown how monostability can be recovered and 
offered an explanation for why multistability has not been seen in previous systems. Finally, we have illustrated a simple procedure to take any four-vertex and transfer all of its stability features into homogeneous stable states of a fold tessellation, thus creating a way to program multistability into the underlying structure of sheetlike materials.

Our work suggests many new and interesting directions. Higher $n$-vertices will lead to much richer single vertex energy landscapes-are more states possible as $n$ increases? We have restricted ourselves to flat systems, but for nonflat systems where $\sum \alpha_{i} \neq 2 \pi$, the branching point disappears - how does the energy landscape change under these circumstances? As pointed out by Schenk and Guest [17], folded tessellations can be stacked-is it possible to make multistable 3D folding materials? To what degree can the energy landscapes be tuned? With increasing complexity, one can imagine that folding planar structures might provide a platform to create metamaterials with arbitrarily tunable mechanical functionality.

We thank I. Cohen, C. Coulais, P. Dieleman, A. Evans, R. Lang, F. Lechenault, C. Santangelo, J. Silverberg, and V. Vitelli for productive discussions. B. G. C. acknowledges support from FOM, and S. W. and M. v. H. acknowledge support from NWO.

[1] M. Kadic, T. Bückmann, R. Schittny, and M. Wegener, Rep. Prog. Phys. 76, 126501 (2013).

[2] R. Lakes, Science 235, 1038 (1987).

[3] G. W. Milton, J. Mech. Phys. Solids 40, 1105 (1992).

[4] M. Kadic, T. Bückmann, N. Stenger, M. Thiel, and M. Wegener, Appl. Phys. Lett. 100, 191901 (2012).

[5] T. Bückmann, M. Thiel, M. Kadic, R. Schittny, and M. Wegener, Nat. Commun. 5, 4130 (2014).

[6] R. S. Lakes, T. Lee, A. Bersie, and Y.C. Wang, Nature (London) 410, 565 (2001).

[7] Z. G. Nicolaou and A. E. Motter, Nat. Mater. 11, 608 (2012).

[8] T. Mullin, S. Deschanel, K. Bertoldi, and M. C. Boyce, Phys. Rev. Lett. 99, 084301 (2007).

[9] K. Bertoldi, M. Boyce, S. Deschanel, S. Prange, and T. Mullin, J. Mech. Phys. Solids 56, 2642 (2008).

[10] K. Bertoldi, P. M. Reis, S. Willshaw, and T. Mullin, Adv. Mater. 22, 361 (2010).
[11] J. T. B. Overvelde, S. Shan, and K. Bertoldi, Adv. Mater. 24, 2337 (2012).

[12] J. Shim, S. Shan, A. Košmrlj, S. Kang, E. R. Chen, J. Weaver, and K. Bertoldi, Soft Matter 9, 8198 (2013).

[13] B. Florijn, C. Coulais, and M. van Hecke, Phys. Rev. Lett. 113, 175503 (2014).

[14] C. L. Kane and T. C. Lubensky, Nat. Phys. 10, 39 (2014).

[15] B. G. Chen, N. Upadhyaya, and V. Vitelli, Proc Natl Acad Sci Unit States Am 111, 13004 (2014).

[16] Z. Y. Wei, Z. V. Guo, L. Dudte, H. Y. Liang, and L. Mahadevan, Phys. Rev. Lett. 110, 215501 (2013).

[17] M. Schenk and S. D. Guest, Proc. Natl. Acad. Sci. U.S.A. 110, 3276 (2013).

[18] J. L. Silverberg, A. A. Evans, L. McLeod, R. C. Hayward, T. Hull, C. D. Santangelo, and I. Cohen, Science 345, 647 (2014).

[19] Lv Cheng, D. Krishnaraju, G. Konjevod, H. Yu, and H. Jiang, Nat. Sci. Rep. 4, 5979 (2014).

[20] B. H. Hanna, J. M. Lund, R. J. Lang, S. P. Magleby, and L. L. Howell, Smart Mater. Struct. 23, 094009 (2014).

[21] E. D. Demaine, M. L. Demaine, V. Hart, G. N. Price, and T. Tachi, Graph Combinator 27, 377 (2011).

[22] This can be seen by cutting one fold - the resulting system has three folding degrees of freedom, two of which are used to close the opened fold, leaving one nontrivial degree of freedom.

[23] E. D. Demaine et al., Geometric Folding Algorithms: Linkages, Origami, Polyhedra (Cambridge University Press, New York, 2007).

[24] S. R. Waitukaitis, R. Menaut, and M. Hecke (to be published).

[25] D. A. Huffman, IEEE Trans. Electron. Comput. C-25, 1010 (1976).

[26] See the Supplemental Material at http://link.aps.org/ supplemental/10.1103/PhysRevLett.114.055503 for movies illustrating folding motions of vertices and sheets.

[27] M. A. Dias, L. H. Dudte, L. Mahadevan, and C. D. Santangelo, Phys. Rev. Lett. 109, 114301 (2012).

[28] F. Lechenault, B. Thiria, and M. Adda-Bedia, Phys. Rev. Lett. 112, 244301 (2014).

[29] C. Coulais, J. T. B. Overvelde, L. A. Lubbers, K. Bertoldi, and M. van Hecke (to be published); J. L. Lubbers and M. van Hecke (to be published)

[30] In our flat sampling of generic vertices, we found one out of $10^{6}$ realizations to be pentastable. By perturbing its parameters, we have found it to persist and to inhabit a finite fraction of parameter space. 